Review

\title{
Resistance profiles to antimicrobial agents in bacteria isolated from acute endodontic infections: systematic review and meta-analysis
}

\author{
Pauline M. Lang a , Rogério C. Jacinto ${ }^{\text {b }}$, Tatiane S. Dal Pizzol c, Maria Beatriz C. Ferreira ${ }^{\text {, }}$, \\ Francisco Montagner ${ }^{\mathrm{e}, *}$ \\ a Federal University of Rio Grande do Sul, Porto Alegre, RS, Brazil \\ ${ }^{\mathrm{b}}$ Endodontic Division, Department of Restorative Dentistry, Univ. Estadual Paulista, Araçatuba, SP, Brazil \\ c Post-Graduation Program in Epidemiology, Faculty of Medicine, Federal University of Rio Grande do Sul, Porto Alegre, Brazil \\ ${ }^{d}$ Department of Pharmacology, Institute of Health Basic Sciences, Federal University of Rio Grande do Sul, Porto Alegre, RS, Brazil \\ e Endodontic Division, Department of Conservative Dentistry, Federal University of Rio Grande do Sul, Rua Ramiro Barcelos, 2492 Bairro Santana, \\ 90035-003 Porto Alegre, RS, Brazil
}

\section{A R T I C L E I N F O}

Article history:

Received 22 March 2016

Accepted 8 August 2016

\section{Keywords:}

Antimicrobial agent

Susceptibility

Acute endodontic infection

Periapical abscess

Meta-analysis

\begin{abstract}
A B S T R A C T
Infected root canal or acute apical abscess exudates can harbour several species, including Fusobacterium, Porphyromonas, Prevotella, Parvimonas, Streptococcus, Treponema, Olsenella and not-yet cultivable species. A systematic review and meta-analysis was performed to assess resistance rates to antimicrobial agents in clinical studies that isolated bacteria from acute endodontic infections. Electronic databases and the grey literature were searched up to May 2015. Clinical studies in humans evaluating the antimicrobial resistance of primary acute endodontic infection isolates were included. PRISMA guidelines were followed. A random-effect meta-analysis was employed. The outcome was described as the pooled resistance rates for each antimicrobial agent. Heterogeneity and sensitivity analyses were performed. Subgroup analyses were conducted based upon report or not of the use of antibiotics prior to sampling as an exclusion factor (subgroups A and B, respectively). Data from seven studies were extracted. Resistance rates for 15 different antimicrobial agents were evaluated (range, 3.5-40.0\%). Lower resistance rates were observed for amoxicillin/clavulanic acid and amoxicillin; higher resistance rates were detected for tetracycline. Resistance rates varied according to previous use of an antimicrobial agent as demonstrated by the subgroup analyses. Heterogeneity was observed for the resistance profiles of penicillin $G$ in subgroup A and for amoxicillin, clindamycin, metronidazole and tetracycline in subgroup B. Sensitivity analyses demonstrated that resistance rates changed for metronidazole, clindamycin, tetracycline and amoxicillin. These findings suggest that clinical isolates had low resistance to $\beta$-lactams. Further welldesigned studies are needed to clarify whether the differences in susceptibility among the antimicrobial agents may influence clinical responses to treatment.
\end{abstract}

(C) 2016 Elsevier B.V. and International Society of Chemotherapy. All rights reserved.

\section{Introduction}

Endodontic infections occur due to caries or dental trauma when opportunistic bacterial pathogens gain access to the necrotic dental pulp or periapical tissues [1,2]. The infected root canal or acute apical abscess can harbour several species, including species belonging to the genera Fusobacterium, Porphyromonas, Prevotella, Parvimonas, Streptococcus, Treponema and Olsenella spp. as well as not-yet cultivable species $[3,4]$. Despite the broad range of species that have been isolated in acute endodontic infections, the microbial profiles

\footnotetext{
* Corresponding author. Endodontic Division, Department of Conservative Dentistry, Federal University of Rio Grande do Sul, Rua Ramiro Barcelos, 2492 Bairro Santana, 90035-003 Porto Alegre, Brazil. Fax: +555133085002.

E-mail address: francisco.montagner@ufrgs.br (F. Montagner).
}

in these communities show few shared species and a great diversity among subjects [5]. Only the strict anaerobes Olsenella profusa and the taxon Dialister E1 were detected in all of the samples analysed by Jacinto et al [6] and Munson et al [7], respectively. However, Tannerella forsythia, Shuttleworthia satelles and Filifactor alocis were only detected in one sample [6]. Interactions among biofilm community members are responsible for the presence of painful symptomatology $[8,9]$. Clinical signs and symptoms have been associated with specific bacterial species: pain with Peptostreptococcus micros, Prevotella intermedia/nigrescens and Eubacterium spp.; tenderness to percussion with Porphyromonas, Peptostreptococcus and Fusobacterium spp.; and swelling with Peptostreptococcus, Porphyromonas and Fusobacterium spp [3].

Clinical management of an acute endodontic infection involves root canal debridement and local drainage, whenever possible. In specific situations, antibiotics may be prescribed as a complementary 
measure, especially for: abscesses that are associated with systemic involvement, including fever, malaise and lymphadenopathy; disseminating infections resulting in cellulitis, progressive diffuse swelling and/or trismus; and abscesses in systemically compromised patients who are at an increased risk of a secondary infection following bacteraemia [2]. The choice of antibiotic is usually based upon previously published susceptibility, testing and clinical trials [1]. The $\beta$-lactam antibiotics, especially penicillin, have been recommended as being the first-line antibiotics because they work well against most causative bacteria and because penicillin has a low incidence of side effects $[10,11]$. Clindamycin has often been recommended in cases of allergy to penicillin or when penicillin has not been effective [10-12]. In the latter clinical situation, $\beta$-lactamase inhibitors such as clavulanic acid in a combination with amoxicillin have also been indicated to extend the spectrum of coverage $[10,11]$.

The emergence of antibiotic-resistant bacterial strains has increased, especially due to excessive and incorrect use of these particular agents [13]. Gomes et al reported an increase in resistance among anaerobic bacteria isolated from primary endodontic infections over a 9-year period in a Brazilian population [14]. Rational prescription of antimicrobial agents must be based on the resistance patterns of the micro-organisms, the characteristics of the patient (immunosuppression, previously reported allergy) and the drug's characteristics (cost, effectiveness, adverse effects). From a microbiological viewpoint, it requires a comprehensive analysis of the resistance profiles among microbial isolates from endodontic infections. Recently, Moraes et al performed a systematic review to describe the presence of resistance genes to antimicrobial agents in oral environments such as saliva, dental biofilm and endodontic infections [15]. However, there is a lack of information regarding whether the microbial isolates from endodontic infections expressing these virulence factors are conveyed as resistance to antimicrobial agents.

Therefore, the aim of this systematic review and meta-analysis was to depict the antimicrobial resistance profiles of bacterial isolates from primary acute endodontic infections as reported in the current literature.

\section{Materials and methods}

\subsection{Focused patient, intervention, comparison and outcome (PICO) question}

A systematic review was performed using the checklist items reported by the Preferred Reporting Items for Systematic Reviews and Meta-Analysis (PRISMA) [16]. The following focused question was developed in accordance with the recognised PICO format: "What are the resistance rates to antimicrobial agents in studies that have isolated bacteria from those patients with acute endodontic infections?'

\subsection{Eligibility criteria}

Clinical studies evaluating the antimicrobial resistance of bacterial isolates in primary acute endodontic infections in humans by disk diffusion or Etest (bioMérieux, Marcy-l'Étoile, France) methods were included in the survey.

\subsection{Search strategy and information sources}

Electronic searches were performed in PubMed, the Cochrane Library (all results), ISI Web of Knowledge, Scopus, LILACS, OpenGrey, SciELO, the CAPES database, the Grey Literature Report, Curtin University, GreyNet International and the Grey Literature Dentistry Database. Hand-searching was independently and extensively

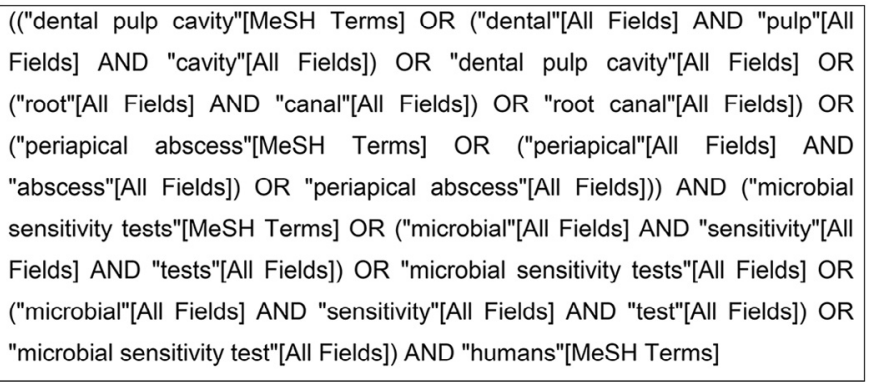

Fig. 1. Search strategy adopted for the study, presenting the MeSH keywords and search terms for antimicrobial activity and the resistance of bacterial isolates from acute endodontic infections, as performed in the PubMed database and adapted for other databases.

performed by two authors (PML and FM) of the reference sections of the selected studies and the available systematic reviews. No language restriction was applied to the search, except for ISI Web of Knowledge. The search comprised those articles published from the inception of the database up to May 2015. Fig. 1 describes the search strategies that were adopted in the study for the PubMed database. This strategy was also employed and adapted for the other databases.

The following limits were used for the ISI Web of Knowledge database: Database (Web of Science ${ }^{\mathrm{TM}}$ Core Collection, Biological Abstracts $^{\circledR}$ and the SciELO Citation Index); Areas of Research (Dentistry and Oral Surgery Medicine, Infectious Diseases, Pharmacology Pharmacy, Microbiology); Document Type (article); and Language (English, Portuguese and Spanish).

\subsection{Study selection and data collection processes}

Following title review and abstract selection, full-text articles were revised based upon the following inclusion criteria: clinical studies in humans that evaluated the antimicrobial resistance of bacterial isolates in primary acute endodontic infections by disk diffusion or Etest methods. Exclusion criteria comprised: (i) studies that did not specify the cause of the odontogenic abscess or the odontogenic infection (whether endodontic or not) or that did not specify the microbial susceptibility results for each source of infection; (ii) studies that did not specify whether the endodontic infection was acute or chronic; and (iii) studies that did not report the method used to evaluate antimicrobial resistance or if another method was used. After reading the included articles, an independent manual search was performed by two of the authors (PML and FM) in the reference section and for the authors of the selected articles.

Data regarding the research group, number of subjects included in the study, description of the recruitment, antibiotic exposure as an exclusion criteria, sample size, methods for sample size determination, conflicts of interest, microbial source/sampling, methods used to measure outcomes, antimicrobial agents tested, statistical analysis, number of bacterial strains and number of resistant strains were collected from all of the studies.

The overall percentage resistance to a specific antimicrobial agent was calculated for each study, regardless of the bacterial species tested. The overall percentage resistance for each tested antimicrobial agent was the average between the total number of resistant strains and the total number of tested strains. Strains that had an intermediate profile were considered susceptible to the antimicrobial agent. According to the National Committee for Clinical Laboratory Standards (NCCLS) [17], the 'intermediate' category included isolates with minimum inhibitory concentrations (MICs) of an antimicrobial agent that approach usually attainable blood and tissue levels and for which response rates may be lower than that 


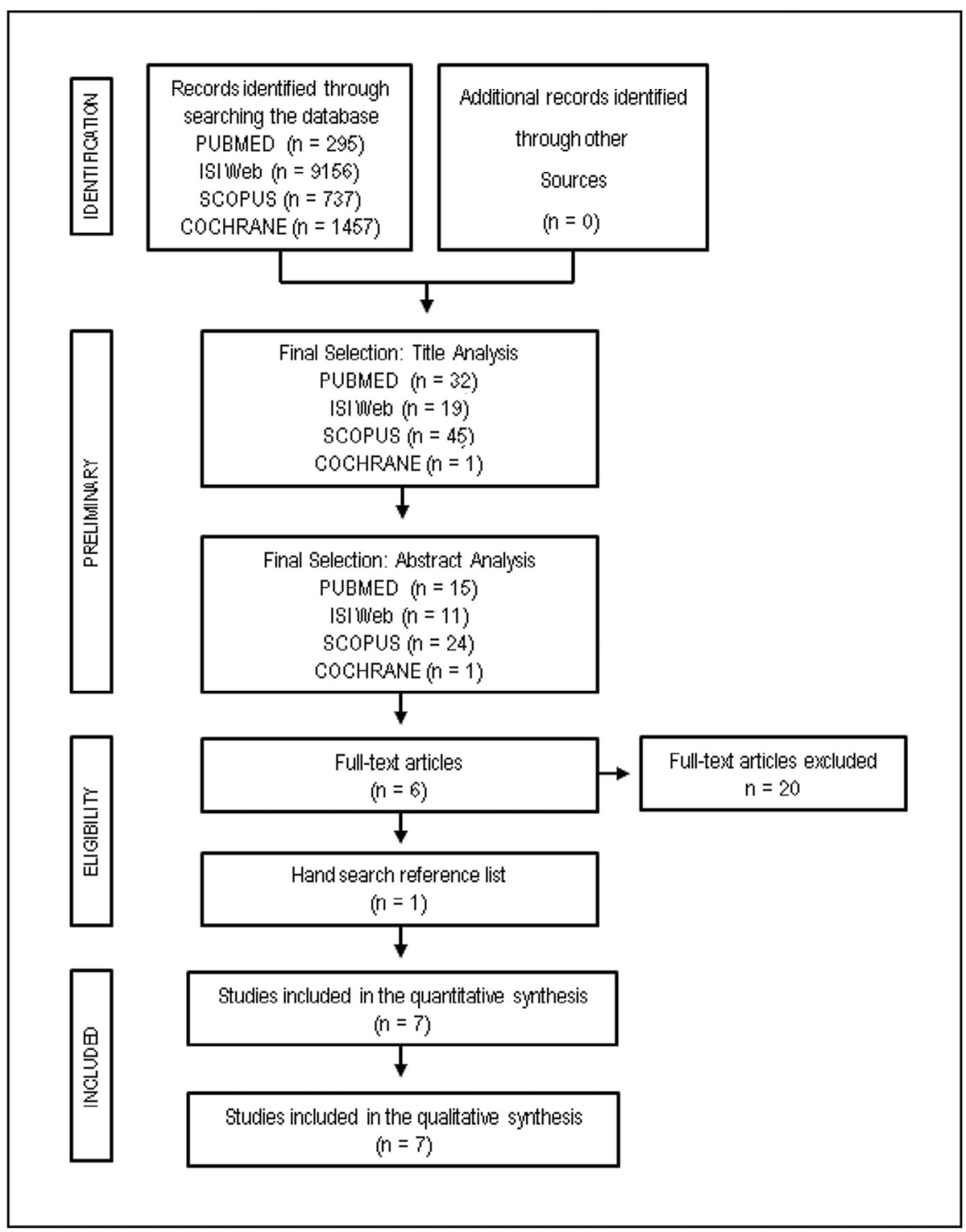

Fig. 2. Flow diagram of the search strategy developed to identify studies related to the antimicrobial resistance of bacterial isolates from acute endodontic infections.

of susceptible isolates. It implies clinical efficacy in body sites where the drug is physiologically concentrated or when a higher than normal dosage of a drug is used. It also includes a buffer zone, which would have prevented small, uncontrolled, technical factors from causing major discrepancies in the interpretations, especially for those drugs with narrow pharmacotoxicity margins.

\subsection{Statistical methods for the meta-analysis}

Statistical analysis was performed to evaluate the resistance profiles of the clinical isolates from primary acute endodontic infections to the antimicrobial agents using Comprehensive Meta-Analysis software v.3.3.070 (Biostat, Englewood, NJ).

A random-effect meta-analysis model was used to estimate the combined effect. The outcome was described as the pooled resistance rates for each antimicrobial agent and was shown using a Forest plot. The degree of heterogeneity was analysed by $\chi^{2}$ test and the $\mathrm{I}^{2}$ statistic, including all of the selected studies for the metaanalysis. A sensitivity analysis was performed by removing those studies with the greatest sample size $[18,19]$ to evaluate the robustness of the results. A subgroup analysis was performed based on the report or not of the use of antibiotics prior to collection as an exclusion factor in the original study. Subgroup A comprised studies that reported the previous use of antimicrobial agents as an exclusion factor [20,21], and Subgroup B included those studies that did not report the previous use of an antimicrobial agent as an exclusion factor $[12,18,19,22,23]$.

\section{Results}

The results of the search strategy are presented in Fig. 2. The final results of the search in The Cochrane Library, ISI Web of Knowledge, Medline database (via PubMed) and Scopus yielded 1, 11, 15 and 24 publications, respectively. Several studies were shared in more than one database (Medline vs. ISI Web of Knowledge, 6; Medline vs. Scopus, 15; and Scopus vs. ISI Web of Knowledge, 9). Implementing the inclusion and exclusion criteria, 6 studies were included and 20 studies were excluded. One additional study was considered relevant by hand-searching.

Information about the selected studies is shown in Tables 1-4. The studies were performed over different time periods (20022014) and the samples were collected in Japan, the USA, Brazil and 
Table 1

Study aims and characteristics of the samples in the included studies.

\begin{tabular}{|c|c|c|c|c|}
\hline Author, year & Aims & Participants & $\begin{array}{l}\text { Antibiotic exposure as } \\
\text { exclusion criterion }\end{array}$ & $\begin{array}{l}\text { Sample } \\
\text { size }^{\mathrm{a}}\end{array}$ \\
\hline $\begin{array}{l}\text { Khemaleelakul et al, } \\
2002[22]\end{array}$ & $\begin{array}{l}\text { To determine the bacterial composition of the microbiota from } \\
\text { acute endodontic abscesses/cellulitis; to determine the } \\
\text { antimicrobial susceptibility of bacteria by Etest }\end{array}$ & 17 patients (age $6-45$ years) & No & 118 \\
\hline Jacinto et al, 2003 [23] & $\begin{array}{l}\text { To investigate the correlation between the composition of } \\
\text { bacterial flora isolated from infected root canals of teeth with } \\
\text { apical periodontitis and with the presence of clinical signs and } \\
\text { symptoms; to test the antibiotic susceptibility of five anaerobic } \\
\text { bacteria most commonly found in the root canals of symptomatic } \\
\text { teeth }\end{array}$ & 48 patients (age $13-63$ years) & No & 66 \\
\hline Kuriyama et al, 2005 [18] & $\begin{array}{l}\text { To determine whether treatment of dentoalveolar infection was } \\
\text { influenced by the choice of antibiotic and the presence of } \\
\text { penicillin-resistant bacteria; to determine any correlation between } \\
\text { the presence of antibiotic resistance within the infection and a } \\
\text { history of previous antibiotic therapy }\end{array}$ & 112 patients (age $17-81$ years) & No & 410 \\
\hline Ozbek et al, 2006 [21] & $\begin{array}{l}\text { To identify micro-organisms in root canals with periapical } \\
\text { abscesses and their antimicrobial susceptibility profiles and to } \\
\text { revise the antimicrobial treatment protocols }\end{array}$ & $\begin{array}{l}30 \text { patients: } \\
14 \text { males (mean age } 31.8 \text { years) } \\
16 \text { females (mean age } 33.8 \text { years) }\end{array}$ & Yes (3 months) & 156 \\
\hline Skucaite et al, 2010 [12] & $\begin{array}{l}\text { To evaluate the susceptibilities of endodontic pathogens isolated } \\
\text { from teeth with symptomatic apical periodontitis to the most } \\
\text { commonly prescribed antibiotics }\end{array}$ & 58 patients (age $20-73$ years) & No & 66 \\
\hline Poeschl et al, 2011 [19] & $\begin{array}{l}\text { To evaluate the actual bacterial resistance rates against the most } \\
\text { commonly used antibiotics and to assess the clinical impact of the } \\
\text { findings }\end{array}$ & 89 patients (age $8-85$ years) & No & 122 \\
\hline Montagner et al, 2014 [20] & $\begin{array}{l}\text { To detect the } c f x A / c f x A 2 \text { gene through molecular methods and to } \\
\text { observe its expression through the MIC and the degradation of a } \\
\text { lactamase substrate }\end{array}$ & $\begin{array}{l}20 \text { patients (average age not } \\
\text { mentioned) }\end{array}$ & Yes (3 months) & 29 \\
\hline
\end{tabular}

MIC, minimum inhibitory concentration.

a Number of bacterial strains tested for antimicrobial susceptibility.

European countries. No selected study mentioned having conducted a sample size calculation; no study described how recruitment was conducted. Two selected papers reported no conflicts of interest $[19,20]$. Samples were collected from the root canals (symptomatic) and apical swellings. The vast majority of studies had not adopted previous exposure to antimicrobial agents as an exclusion criterion. Descriptive statistics were reported in all publications.

Data from seven studies were extracted [12,18-23]. A total of 15 different antimicrobial agents were evaluated in the selected studies, as follows: penicillin; amoxicillin; amoxicillin/clavulanic acid (AMC); ampicillin; piperacillin/tazobactam (TZP); clindamycin; metronidazole; erythromycin; azithromycin; cefaclor; cefazolin; cefoxitin; vancomycin; imipenem; and tetracycline. Results of the metaanalysis are shown in Fig. 3. The data are summarised in Table 5.

The overall resistance rates ranged from $3.5 \%$ to $40.0 \%$ for microorganisms isolated from acute endodontic infections (Table 5). After the sensitivity analysis excluding the study of Kuriyama et al [18], the overall resistance rates were $9.5 \%, 23.2 \%, 25.4 \%$ and $64 \%$ of isolates for metronidazole, erythromycin, clindamycin and tetracycline, respectively. After the sensitivity analysis excluding the study of Poeschel et al [19], the overall resistance rates were $4.9 \%, 4.3 \%$ and $12.9 \%$ of isolates to amoxicillin, AMC and penicillin G, respectively.

Subgroup analysis showed that the resistance rates ranged from $6.9 \%$ to $82.9 \%$ for studies that reported previous use of antimicrobial agents as an exclusion factor in the original study. However, they ranged from $1.4 \%$ to $21.7 \%$ for studies that did not report or did not employ previous use of antimicrobial agents as an exclusion factor.

The study observed heterogeneity in the resistance rates for penicillin $G(Q$-value $=9.479 ; P=0.002)$ among the studies that reported previous use of antimicrobial agents as an exclusion factor (subgroup A). The same behaviour was not observed for AMC (Qvalue $=3.011 ; P=0.083$ ). In subgroup $A$, heterogeneity analysis was not performed for clindamycin, erythromycin, metronidazole, tetracycline and amoxicillin because only one study included them (Fig. 3A). Heterogeneity was also observed for amoxicillin (Qvalue $=9.809 ; P=0.02)$, clindamycin $(Q$-value $=43.906 ; P=0.000)$, metronidazole $(\mathrm{Q}$-value $=15.536 ; P=0.001)$ and tetracycline $(\mathrm{Q}$ value $=33.7 ; P=0.000$ ) among those studies that had not reported the previous use of antimicrobial agents as being an exclusion factor

Table 2

Other information in the included studies.

\begin{tabular}{|c|c|c|c|}
\hline Author, year & Microbial source/sampling & $\begin{array}{l}\text { Method used to } \\
\text { measure outcome }\end{array}$ & Antimicrobial agents \\
\hline Khemaleelakul et al, 2002 [22] & Tissue swelling/aspiration & Etest & Penicillin; amoxicillin; amoxicillin/clavulanic acid; clindamycin; metronidazole \\
\hline Jacinto et al, 2003 [23] & Root canal/paper points & Etest & $\begin{array}{l}\text { Penicillin; amoxicillin; amoxicillin/clavulanic acid; clindamycin; metronidazole; } \\
\text { erythromycin; cefaclor; azithromycin }\end{array}$ \\
\hline Kuriyama et al, 2005 [18] & $\begin{array}{l}\text { Root canal/swab } \\
\text { Tissue swelling/aspiration }\end{array}$ & Disk diffusion & Penicillin; clindamycin; metronidazole; erythromycin; tetracycline \\
\hline Ozbek et al, 2006 [21] & Root canal/paper points & Oxoid disks and Etest & $\begin{array}{l}\text { Penicillin; amoxicillin/clavulanic acid; clindamycin; erythromycin; tetracycline; } \\
\text { cefazolin; imipenem; metronidazole; cefoxitin; piperacillin/tazobactam }\end{array}$ \\
\hline Skucaite et al, 2010 [12] & $\begin{array}{l}\text { Root canal/paper points } \\
\text { Tissue swelling/aspiration }\end{array}$ & Etest & $\begin{array}{l}\text { Penicillin; amoxicillin; amoxicillin/clavulanic acid; clindamycin; metronidazole; } \\
\text { erythromycin; tetracycline; ampicillin; vancomycin }\end{array}$ \\
\hline Poeschl et al, 2011 [19] & $\begin{array}{l}\text { Tissue swelling/swabbing } \\
\text { or aspiration }\end{array}$ & Disk diffusion & Penicillin; amoxicillin; amoxicillin/clavulanic acid; clindamycin; erythromycin \\
\hline Montagner et al, 2014 [20] & Root canal/paper points & Etest, Nitrocefin test & Penicillin; amoxicillin; amoxicillin/clavulanic acid \\
\hline
\end{tabular}


Table 3

Genera of tested bacteria that were reported in each included study.

\begin{tabular}{|c|c|}
\hline Author, year & Bacterial genera \\
\hline Khemaleelakul et al, 2002 [22] & $\begin{array}{l}\text { Bacteroides, Clostridium, Eubacterium, Fusobacterium, Peptostreptococcus, Porphyromonas, Prevotella, Veillonella, Propionibacterium, } \\
\text { Actinomyces, Gemella, Streptococcus, Corynebacterium, Eikenella, Lactobacillus, Staphylococcus }\end{array}$ \\
\hline Jacinto et al, 2003 [23] & $\begin{array}{l}\text { Fusobacterium, Peptostreptococcus, Streptococcus, Prevotella, Gemella, Actinomyces, Veillonella, Clostridium, Propionibacterium, } \\
\text { Eggerthella, Staphylococcus, Eubacterium, Enterococcus, Campylobacter, Bifidobacterium, Bacteroides, Tissierella, Lactobacillus, } \\
\text { Porphyromonas }\end{array}$ \\
\hline Kuriyama et al, 2005 [18] & $\begin{array}{l}\text { Prevotella, Peptostreptococcus, Streptococcus, Fusobacterium, Eubacterium, Actinomyces, Eikenella, Veillonella, Propionibacterium, } \\
\text { Porphyromonas, Capnocytophaga, Clostridium, unspecified, strictly anaerobic Gram-negative bacillus, unidentified } \mathrm{CO}_{2} \text {-dependent } \\
\text { Gram-positive coccus }\end{array}$ \\
\hline Ozbek et al, 2006 [21] & $\begin{array}{l}\text { Staphylococcus, Streptococcus, Corynebacterium, Neisseria, Acinetobacter, Escherichia, Pseudomonas, Enterobacter, Klebsiella, } \\
\text { Peptostreptococcus, Actinomyces, Eubacterium, Mobiluncus, Erysipelothrix, Fusobacterium, Prevotella, Bacteroides, Porphyromonas }\end{array}$ \\
\hline Skucaite et al, 2010 [12] & Streptococcus, Enterococcus, Prevotella, Bacteroides, Anaerococcus, Peptostreptococcus, Tissierella, Eikenella \\
\hline Poeschl et al, 2011 [19] & $\begin{array}{l}\text { Streptococcus, Staphylococcus, Prevotella, Peptostreptococcus, Bacteroides, Fusobacterium, Eikenella, Lactobacillus, Corynebacterium, } \\
\text { Enterococcus, Enterobacteriaceae }\end{array}$ \\
\hline Montagner et al, 2014 [20] & Prevotella, Porphyromonas, Parvimonas \\
\hline
\end{tabular}

Table 4

Number of resistant strains for each antibiotic in the included studies.

\begin{tabular}{|c|c|c|c|c|c|c|c|c|c|c|c|c|c|c|c|c|}
\hline \multirow[t]{2}{*}{ Author, year } & \multirow{2}{*}{$\begin{array}{l}\text { No. of bacterial } \\
\text { strains tested }\end{array}$} & \multicolumn{15}{|c|}{ No. of resistant strains } \\
\hline & & PCG & PCV & AMX & AMC & CLI & MTZ & ERY & TET & CEZ & $\mathrm{CCL}$ & AMP & VAN & IPM & CFX & TZP \\
\hline \multirow[t]{2}{*}{ Khemaleelakul et al, 2002 [22] } & 118 & - & 23 & 18 & 0 & 13 & - & - & - & - & - & - & - & - & - & - \\
\hline & 58 & - & - & - & - & - & 7 & - & - & - & - & - & - & - & - & - \\
\hline Jacinto et al, 2003 [23] & 66 & 7 & - & 0 & 0 & 3 & 3 & 7 & - & - & 0 & - & - & - & - & - \\
\hline Kuriyama et al, 2005 [18] & 410 & - & 53 & - & - & 13 & 82 & 96 & 36 & - & - & - & - & - & - & - \\
\hline \multirow[t]{3}{*}{ Ozbek et al, 2006 [21 $]^{\mathrm{a}}$} & 156 & 74 & - & - & - & 57 & - & - & - & - & - & - & - & - & - & - \\
\hline & 76 & - & - & - & 17 & - & 15 & 39 & 63 & 23 & - & - & - & - & - & - \\
\hline & 70 & - & - & - & - & - & - & - & - & - & - & - & - & 3 & 14 & 7 \\
\hline \multirow[t]{5}{*}{ Skucaite et al, 2010 [12] } & 66 & - & - & - & - & - & - & - & - & - & - & 21 & 0 & - & - & - \\
\hline & 65 & 1 & - & - & - & 14 & - & - & - & - & - & - & - & - & - & - \\
\hline & 53 & - & - & 0 & - & - & - & 15 & 21 & - & - & - & - & - & - & - \\
\hline & 10 & - & - & - & 0 & - & - & - & - & - & - & - & - & - & - & - \\
\hline & 9 & - & - & - & - & - & 5 & - & - & - & - & - & - & - & - & - \\
\hline \multirow[t]{4}{*}{ Poeschl et al, 2011 [19] } & 122 & - & - & 11 & 2 & - & - & - & - & - & - & - & - & - & - & - \\
\hline & 107 & - & - & - & - & 23 & - & - & - & - & - & - & - & - & - & - \\
\hline & 103 & 10 & - & - & - & - & - & - & - & - & - & - & - & - & - & - \\
\hline & 76 & - & - & - & - & - & - & 17 & - & - & - & - & - & - & - & - \\
\hline Montagner et al, $2014[20]^{a}$ & 29 & 4 & - & 2 & 2 & - & - & - & - & - & - & - & - & - & - & - \\
\hline
\end{tabular}

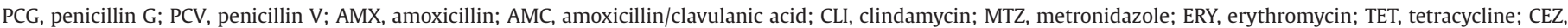
cefazolin; CCL, cefaclor; AMP, ampicillin; VAN, vancomycin; IPM, imipenem; CFX, cefoxitin; TZP, piperacillin/tazobactam.

-, not tested.

a Original studies that reported prior use of antibiotics as an exclusion criterion.

(subgroup B). Penicillin G (Q-value $=3.519 ; P=0.172)$, penicillin $\mathrm{V}$ $(\mathrm{Q}$-value $=3.161 ; P=0.075)$, AMC $(\mathrm{Q}$-value $=1.683 ; P=0.641)$ and erythromycin $(Q-v a l u e=6.204 ; P=0.102)$ did not show heterogeneity among resistance rates in the articles belonging to subgroup $B$ that did not report the previous use of antimicrobial agents as being an exclusion factor (Fig. 3B).

A random-effect meta-analysis model was not performed for cefaclor, tetracycline, cefazolin, ampicillin, azithromycin, vancomycin, imipenem, cefoxitin and TZP because they were mentioned in only a single study [21]. The resistance rates to these antimicrobial agents are shown in Table 4 .

\section{Discussion}

In the present study, a comprehensive systematic review was conducted to identify, evaluate and synthesise all of the clinical studies that met the specified eligibility criteria in order to determine: "What are the resistance rates to antimicrobial agents in studies that isolated bacteria from patients with primary acute endodontic infections?' Although the majority of clinical isolates have been found to be susceptible to the antimicrobial agents that are usually prescribed, there was a wide range of antibiotic resistance among them. The resistance rates varied according to the previous use of an antimicrobial agent.
The currently employed methods to determine the susceptibility profiles of clinical isolates are regulated by standard protocols that employ a cultivation-based approach $[17,24]$. Only articles that employed the disk diffusion and Etest methods were selected because they included patterns that allow for a comparison according to NCCLS and European Committee on Antimicrobial Susceptibility Testing (EUCAST) guidelines. Continuous monitoring of microbial susceptibility over time should be encouraged, but there are few reports in the current literature that describe the shift in antimicrobial resistance in bacteria isolated from endodontic infections [14]. There is a need for constantly revising the literature in order to obtain proper data that may guide and support the clinical choices of adjunctive systemic antimicrobial therapy. Use of statistical methods, as performed in the meta-analysis, may provide a quantitative synthesis of the data regarding the susceptibility rates of clinical isolates from patients with primary acute endodontic infections.

Despite the strict selection criteria, evaluation of the studies showed few factors that connected them. For example, several species were isolated and tested for their antimicrobial susceptibility. Furthermore, the included articles did not test the same set of antimicrobial agents for susceptibility of all of the isolates. The little amount of shared information in the studies did not allow for the determination through a meta-analysis of the resistance 
(A)

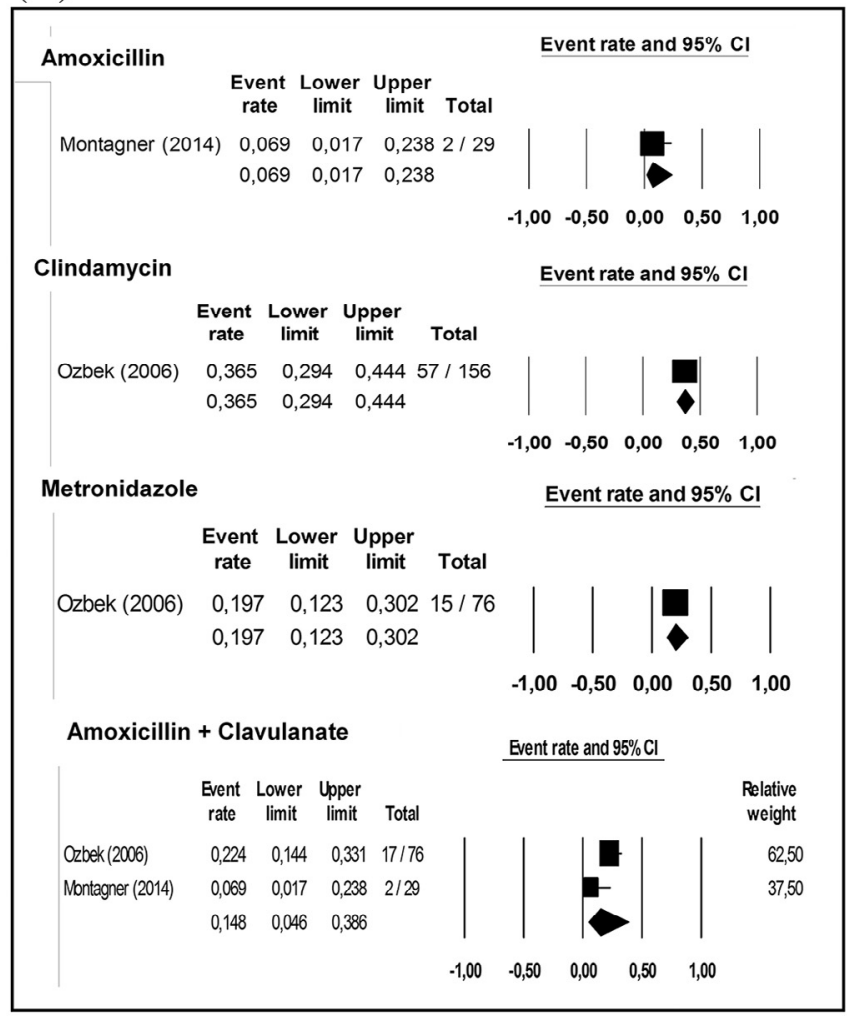

(B)

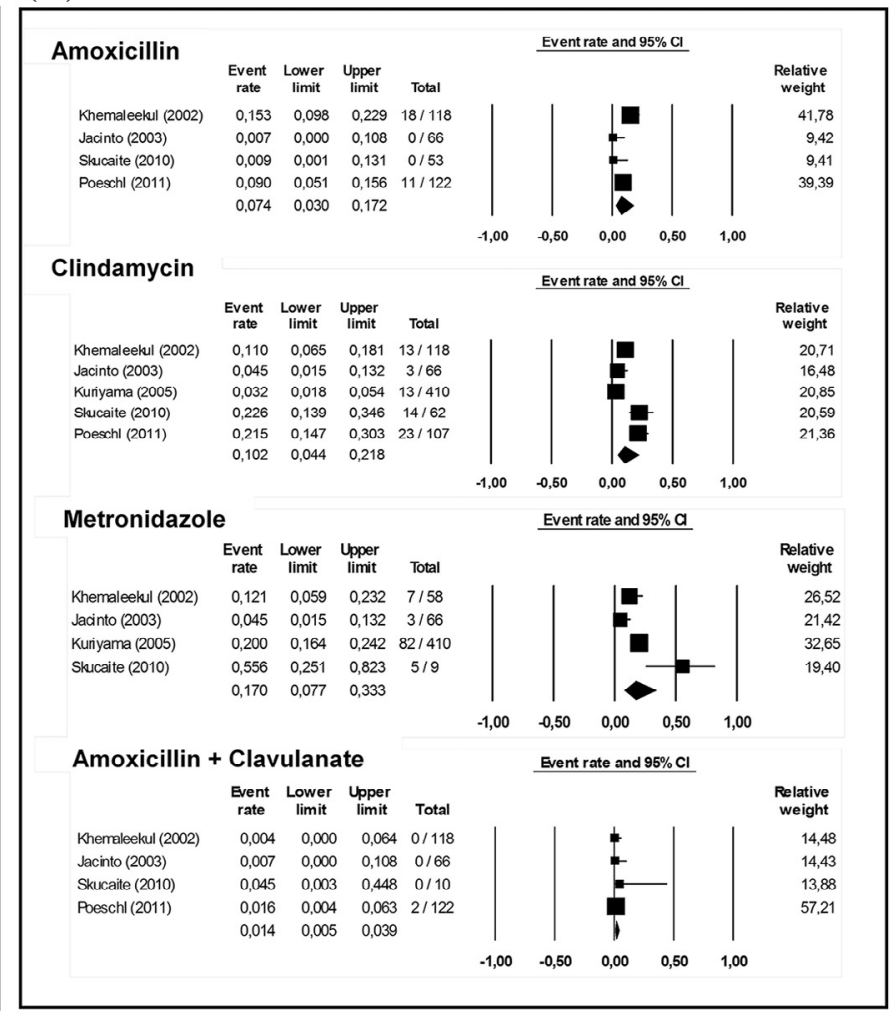

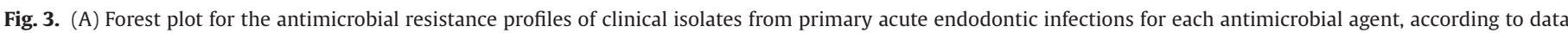

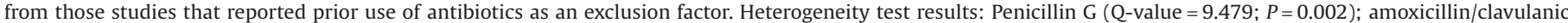

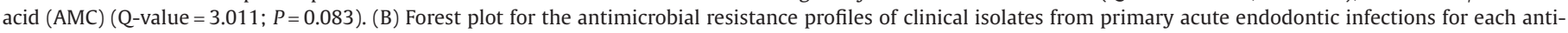

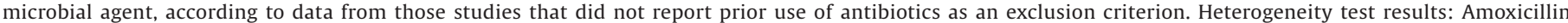

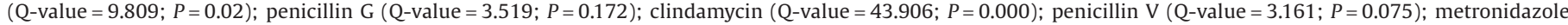

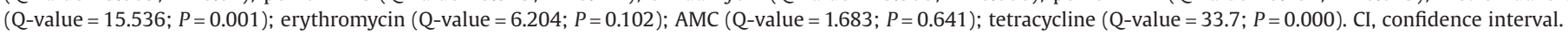

profiles for each species/genera. The overall resistance percentages for a specific antimicrobial agent were calculated for each study, regardless of the species that were tested.

For primary acute endodontic infections, the overall resistance rates varied according to the antimicrobial agent. The bacterial strains were highly susceptible to AMC and amoxicillin. Higher resistance rates were observed for tetracycline. Intermediate values were observed for penicillin $\mathrm{G}$, clindamycin, penicillin $\mathrm{V}$, metronidazole and erythromycin. The results were in accordance with clinical recommendations which suggest antibiotics of the $\beta$-lactam group, especially amoxicillin and AMC, as being the first choice for the management of acute endodontic infections owing to their efficacy, safety, and convenience for administration and access $[10,25,26]$. The low susceptibility rates for tetracycline were probably associated with intrinsic resistance among the anaerobic strains as well as secondary resistance due to its broad use and misuse [27,28]. For the past two decades, the most commonly used antibiotics in periodontal treatment have been the tetracyclines [29,30]. The widespread emergence of tetracycline resistance in medically important bacteria has limited the use of tetracycline in the treatment of medical infections [31]. In the oral cavity, tetracycline resistance has increased over

Table 5

The pooled resistance rate, as determined by data extracted from the included studies.

\begin{tabular}{|c|c|c|c|c|c|c|c|c|c|}
\hline \multirow[t]{2}{*}{ Antibiotic } & \multicolumn{3}{|c|}{ Overall data } & \multicolumn{3}{|c|}{$\begin{array}{l}\text { Studies that reported previous } \\
\text { use of ATBs as exclusion } \\
\text { criterion (subgroup A) }\end{array}$} & \multicolumn{3}{|c|}{$\begin{array}{l}\text { Studies that did not report/employ } \\
\text { previous use of ATBs as exclusion } \\
\text { criterion (subgroup B) }\end{array}$} \\
\hline & $\%_{\text {RES }}{ }^{b}$ & $\mathrm{CI}$ & $n_{\mathrm{RES}} / n^{\mathrm{c}}$ & $\%$ RES $^{\mathrm{b}}$ & $\mathrm{CI}$ & $n_{\mathrm{RES}} / n^{\mathrm{c}}$ & $\%_{\mathrm{RES}}^{\mathrm{b}}$ & $\mathrm{CI}$ & $n_{\mathrm{RES}} / n^{\mathrm{c}}$ \\
\hline AMC & 3.5 & $0.8-14.2$ & $21 / 421$ & 14.8 & $4.6-38.6$ & $19 / 105$ & 1.4 & $0.5-3.9$ & $2 / 316$ \\
\hline Amoxicillin & 7.7 & $3.6-15.5$ & $31 / 388$ & 6.9 & $1.7-23.8$ & $2 / 29$ & 7.4 & $3.0-17.2$ & $29 / 359$ \\
\hline Penicillin G & 12.3 & $3.6-34.6$ & $100 / 458$ & 29.1 & $7.0-68.9$ & $78 / 185$ & 7.9 & $4.0-15.0$ & $18 / 357$ \\
\hline Clindamycin & 13.1 & $5.6-27.5$ & $123 / 919$ & 36.5 & $29.4-44.4$ & $57 / 156$ & 10.2 & $4.4-21.8$ & $66 / 756$ \\
\hline Penicillin V & 15.5 & $10.2-22.8$ & $76 / 528$ & - & - & - & 15.5 & $10.2-22.8$ & $76 / 528$ \\
\hline Metronidazole & 17.5 & $10.5-27.9$ & $112 / 619$ & 19.7 & $12.3-30.2$ & $15 / 76$ & 17.0 & $7.7-33.3$ & $97 / 543$ \\
\hline Erythromycin & 26.0 & $16.2-38.9$ & $174 / 681$ & 51.3 & $40.2-62.3$ & $39 / 76$ & 21.7 & $16.2-28.3$ & $135 / 605$ \\
\hline Tetracycline & 40.0 & $6.2-87.0$ & $120 / 539$ & 82.9 & $72.7-89.8$ & $63 / 76$ & 19.9 & $3.6-61.9$ & $57 / 463$ \\
\hline
\end{tabular}

ATB, antibiotics; CI, confidence interval; AMC, amoxicillin/clavulanic acid.

a Subgroup A [20,21]; subgroup B [12,18,19,22,23].

b \%RES = percentage of resistant strains (pooled values).

c $n_{\mathrm{RES}} / n$, number of resistant strains/total number of strains. 
the past several years [32]. Some investigators have reported that this may be one of the reasons for the reduced effects of tetracyclines as an adjuvant measure for the treatment of periodontitis [33-36].

Despite the differences in laboratorial susceptibility patterns observed for the antimicrobial agents, it was not possible to determine whether they influence clinical responses to treatment. It should be emphasised that acute endodontic infections are polymicrobial with very high interindividual variability.

The involvement of species in a mixed consortium promotes a broad range of relationships among them, modulating their pathogenicity as additive or synergistic pathogenic effects [2,4]. The control of endodontic infections does not have the species as a main target. Eradication of some components of the microbial community may lead to its disturbance and the remaining members may not be able to survive without cross-interactions [4]. The ecological interference that is promoted by the local approach (such as root canal treatment and surgical drainage) might overcome the resistance to antimicrobial agents as demonstrated by some of the community members and lead to death of the resistant strain. However, Flynn et al reported in a prospective study that penicillin treatment failure was not predicted by pre-admission, timing, anatomic or preoperative clinical variables in a sample of 37 subjects admitted for severe odontogenic infections [37]. The authors observed that 6 of 24 subjects had penicillin treatment failure, and penicillin-resistant bacteria were detected in all of the patients who had no response to the antimicrobial treatment. Therefore, further studies with large sample sizes should be conducted to determine the relationship between the presence of resistant strains and the outcomes of the proposed treatment.

Previous use of antimicrobial agents is a variable that must be considered in the data analysis. Skucaite et al evaluated the susceptibility of endodontic pathogens to antibiotics in patients with symptomatic apical periodontitis who had previous use of an antimicrobial agent [12]. According to the results, no correlation was found between microbial susceptibility to antibiotics and previous antibiotic intake. However, Kuriyama et al showed that penicillinresistant bacteria were isolated more frequently from patients who had received penicillin before sampling [18]. There was no significant correlation between the prevalence of erythromycin-resistant bacteria and previous administration of erythromycin. Therefore, a subgroup analysis was performed regarding the results to determine the effects of previous use of antibiotics. It was observed that the resistance rates were different when comparing studies that reported previous use of antimicrobial agents as an exclusion criterion (subgroup A). Articles that did not report previous use of antimicrobial agents as an exclusion criterion comprised subgroup B. These differences could be related to diverse factors: the small number of studies; the reduced number of bacterial strains that were tested in each study; the fact that the resistance rates were obtained from a pool of bacteria; and the fact that the correlation between the prevalence of resistant bacteria and previous use of antibiotics appeared to occur for some agents, but not for others. In addition, there was a limitation of memory bias, since patients could not remember exactly what drugs they had used in the past 3 months. These results emphasise the need to analyse the data reported in articles and to consider the previous use or not of antibiotics, since the resistance rates differed between the subgroups. However, independent of the differences among the subgroups A and B and the overall data, the general profiles of resistance remained the same: lower rates of resistance for amoxicillin and AMC; higher rates of resistance for the tetracyclines; and intermediate rates for the other antimicrobial agents that were tested.

In parallel, a heterogeneity analysis was performed for specific antimicrobial agents, depending on the subgroup of the studies. There was heterogeneity among the resistance profiles for penicillin $G$ for the studies that excluded previous use an antimicrobial agent (subgroup A) and for amoxicillin, clindamycin, metronidazole and tetracycline among the studies that did not exclude this use (subgroup B). These findings can be associated with several bacterial species that were tested and for the different sites of sampling. Despite being concomitant infections, matched samples taken from the root canal and abscess aspirates from the same subject had discrepancies between the bacterial community profiles [5]. Furthermore, the microbial profiles of the acute endodontic infection samples were unique for each subject and did not show any clustering behaviour from the samples that were collected among patients $[5,38]$. Despite the assessment of a specific group of endodontic infections, the microbiome was modulated by its geographic location [39-41]. It was not possible to perform a region-based analysis because the samples were collected from six different countries (Thailand, Brazil, UK, Turkey, Lithuania and Austria). Only the results reported by Jacinto et al [23] and Montagner et al [20] belonged to the same geographic location (Brazil).

Sensitivity analyses were performed excluding those studies with the largest sample sizes $[18,19]$ to evaluate whether the findings were dependent on arbitrary or unclear decisions. The overall resistance rates to erythromycin, AMC and penicillin G were similar when the studies were included in the meta-analysis. However, the overall resistance rates to metronidazole, clindamycin, tetracycline and amoxicillin were different when these studies were included in the meta-analysis. Although different, it was not possible to determine whether the difference influenced the clinical response to treatment, as discussed previously.

The results of this systematic review and meta-analysis are limited by several factors, as previously discussed. They are also associated with the limitations that were imposed by bacterial recovery from the complex microbial communities. It should be emphasised that available cultivation methods have not yet been able to allow for the laboratorial growth and isolation of several micro-organisms. Furthermore, the results obtained through susceptibility testing represented a single micro-organism or a select group of microorganisms that were isolated from the infected site. The virulence of the strains might have been modulated by their isolation in the culture medium, especially due to the lack of interaction among the several micro-organisms that were also present in the odontogenic infection. There was also a lack of data on the specific number of strains that were tested for each sample in the articles. The tested isolates may or may not have represented all of the microbial community that was active in the infected site. As culturing and testing of slow-growing oral bacteria can take up to 2 weeks, therapeutic decisions still have to be based on previous reports from the literature, as has been summarised in the present study.

\section{Conclusion}

This systematic review and meta-analysis allowed for depicting the resistance profiles to antimicrobial agents in bacteria isolated from acute endodontic infections. AMC and amoxicillin showed the lowest in vitro rates for bacterial resistance among the strains. The resistance rates for the antimicrobial agents varied according to the previous use of an antimicrobial agent. There was a lack of information regarding the association between the resistance profiles of the bacterial isolates and clinical outcomes.

Funding: None.

Competing interests: None declared.

Ethical approval: Not required.

\section{References}

[1] Baumgartner JC, Xia T. Antibiotic susceptibility of bacteria associated with endodontic abscesses. J Endod 2003;29:44-7. 
[2] Siqueira JF Jr, Rôças IN. Microbiology and treatment of acute apical abscesses. Clin Microbiol Rev 2013;26:255-73.

[3] Gomes BPFA, Pinheiro ET, Gadê-Neto CR, Sousa ELR, Ferraz CCR, Zaia AA, et al. Microbiological examination of infected dental root canals. Oral Microbiol Immunol 2004;19:71-6.

[4] Siqueira JF Jr, Rôças IN. The microbiota of acute apical abscesses. J Dent Res 2009;88:61-5.

[5] Montagner F, Gomes BP, Kumar PS. Molecular fingerprinting reveals the presence of unique communities associated with paired samples of root canals and acute apical abscesses. J Endod 2010;36:1475-9.

[6] Jacinto RC, Gomes BP, Desai M, Rajendram D, Shah HN. Bacterial examination of endodontic infections by clonal analysis in concert with denaturing highperformance liquid chromatography. Oral Microbiol Immunol 2007;22:403-10.

[7] Munson MA, Pitt-Ford T, Chong B, Weightman A, Wade WG. Molecular and cultural analysis of the microflora associated with endodontic infections. J Dent Res 2002;81:761-6.

[8] Siqueira JF Jr, Rôças IN, Rosado AS. Investigation of bacterial communities associated with asymptomatic and symptomatic endodontic infections by denaturing gradient gel electrophoresis fingerprinting approach. Oral Microbiol Immunol 2004; 19:363-70.

[9] Sakamoto M, Rôças IN, Siqueira JF Jr, Benno Y. Molecular analysis of bacteria in asymptomatic and symptomatic endodontic infections. Oral Microbiol Immunol 2006;21:112-22.

[10] Oberoi SS, Dhingra C, Sharma G, Sardana D. Antibiotics in dental practice: how justified are we. Int Dent J 2015;65:4-10.

[11] Holmes CJ, Pellecchia R. Antimicrobial therapy in management of odontogenic infections in general dentistry. Dent Clin North Am 2016;60:497-507.

[12] Skucaite N, Peciuliene V, Vitkauskiene A, Machiulskiene V. Susceptibility of endodontic pathogens to antibiotics in patients with symptomatic apical periodontitis. J Endod 2010;36:1611-16.

[13] Cantas L, Shah SQ, Cavaco LM, Manaia CM, Walsh F, Popowska M, et al. A brief multi-disciplinary review on antimicrobial resistance in medicine and its linkage to the global environmental microbiota. Front Microbiol 2013;14:4-96.

[14] Gomes BPFA, Jacinto RC, Montagner F, Souza ELR, Ferraz CCR. Analysis of the antimicrobial susceptibility of anaerobic bacteria isolated from endodontic infections in Brazil during a period of nine years. J Endod 2011:37:1058-62.

[15] Moraes LC, Só MVR, Dal Pizzol TS, Ferreira MBC, Montagner F. Distribution of genes related to antimicrobial resistance in different oral environments: a systematic review. J Endod 2015;41:434-41.

[16] Moher D, Shamseer L, Clarke M, Ghersi D, Liberati A, Petticrew M, et al. PRISMA-P group. Preferred reporting items for systematic review and metaanalysis protocols (PRISMA-P) 2015 statement. Syst Rev 2015;4:1.

[17] National Committee for Clinical Laboratory Standards. Methods for antimicrobial susceptibility testing of anaerobic bacteria. 6th ed. Wayne, PA: NCCLS; 2004 Document M11-A6.

[18] Kuriyama T, Absi EG, Williams DW, Lewis MAO. An outcome audit of the treatment of acute dentoalveolar infection: impact of penicillin resistance. $\mathrm{Br}$ Dent J 2005;198:759-63.

[19] Poeschl PW, Crepaz V, Russmueller G, Seemann R, Hirschl AM, Ewers R. Endodontic pathogens causing deep neck space infections: clinical impact of different sampling techniques and antibiotic susceptibility. J Endod 2011;37:1201-5.

[20] Montagner F, Jacinto RC, Signoretti FGC, Mattos VS, Grecca FS, Gomes BPFA. $\beta$-Lactamic resistance profiles in Porphyromonas, Prevotella, and Parvimonas species isolated from acute endodontic infections. J Endod 2014;40:339-44.

[21] Ozbek SM, Evcil MS, Ozbek A, Erdogan AS, Köseoglu M. Microbiological analysis of root canals associated with periapical abscesses (with pain and swelling) and the antimicrobial susceptibility of isolated bacteria. Pain Clin 2006;18:32737.

[22] Khemaleelakul S, Baumgartner JC, Pruksakorn S. Identification of bacteria in acute endodontic infections and their antimicrobial susceptibility. Oral Surg Oral Med Oral Pathol Oral Radiol Endod 2002:94:746-55.

[23] Jacinto RC, Gomes BPFA, Ferraz CCR, Zaia AA, Souza Filho FJ. Microbiological analysis of infected root canals from symptomatic and asymptomatic teeth with periapical periodontitis and the antimicrobial susceptibility of some isolated anaerobic bacteria. Oral Microbiol Immunol 2003;18:285-92.

[24] European Committee on Antimicrobial Susceptibility Testing. Disk diffusion test methodology (v.5.0, January 2015), http://www.eucast.org/ast_of_bacteria/ disk_diffusion_methodology/; 2015 [accessed 19.09.16].

[25] Ellison SJ. The role of phenoxymethylpenicillin, amoxicillin, metronidazole and clindamycin in the management of acute dentoalveolar abscesses-a review. Br Dent J 2009;206:357-62.

[26] Poveda Roda R, Bagan JV, Sanchis Bielsa JM, Carbonell Pastor E. Antibiotic use in dental practice. A review. Med Oral Patol Oral Cir Bucal 2007;12:E186-92.

[27] Rodrigues RM, Gonçalves C, Souto R, Feres-Filho EJ, Uzeda M, Colombo AP. Antibiotic resistance profile of the subgingival microbiota following systemic or local tetracycline therapy. J Clin Periodontol 2004;31:420-7.

[28] Roberts MC. Tetracycline therapy: update. Clin Infect Dis 2003;36:462-7.

[29] Van Winkelhoff AJ, Rams TE, Slots J. Systemic antibiotic therapy in periodontics. Periodontol 2000 1996;10:45-78.

[30] Walker C, Karpinia K. Rationale for use of antibiotics in periodontics. J Periodontol 2002;73:1188-96.

[31] Speer BS, Shoemaker NB, Salyers AA. Bacterial resistance to tetracycline: mechanisms, transfer, and clinical significance. Clin Microbiol Rev 1992;5:38799.

[32] Walker CB. The acquisition of antibiotic resistance in the periodontal microflora. Periodontol 2000 1996;10:79-88.

[33] Asikainen S, Jousimies-Somer H, Kanervo A, Saxén L. The immediate efficacy of adjunctive doxycycline in treatment of localized juvenile periodontitis. Arch Oral Biol 1990;35(Suppl.):231S-4S.

[34] Mombelli A, Tonetti M, Lehmann B, Lang NP. Topographic distribution of black-pigmenting anaerobes before and after periodontal treatment by local delivery of tetracycline. J Clin Periodontol 1996;23:906-13.

[35] Colombo AP, Haffajee AD, Dewhirst FE, Paster BJ, Smith CM, Cugini MA, et al. Clinical and microbiological features of refractory periodontitis subjects. J Clin Periodontol 1998;25:169-80.

[36] Wong MY, Lu CL, Liu CM, Hou LT. Microbiological response of localized sites with recurrent periodontitis in maintenance patients treated with tetracycline fibers. J Periodontol 1999; 70:861-8.

[37] Flynn TR, Shanti RM, Hayes C. Severe odontogenic infections, part 2: prospective outcomes study. J Oral Maxillofac Surg 2006;64:1104-13.

[38] Montagner F, Jacinto RC, Signoretti FGC, Sanches PF, Gomes BPFA. Clustering behavior in microbial communities from acute endodontic infections. J Endod 2012;38:158-62.

[39] Rôças IN, Siqueira JF Jr, Debelian GJ. Analysis of symptomatic and asymptomatic primary root canal infections in adult Norwegian patients. J Endod 2011;37:1206-12.

[40] Machado de Oliveira JC, Siqueira JF Jr, Rôças IN, Baumgartner JC, Xia T, Peixoto RS, et al. Bacterial community profiles of endodontic abscesses from Brazilian and USA subjects as compared by denaturing gradient gel electrophoresis analysis. Oral Microbiol Immunol 2007;22:14-18.

[41] Rôças IN, Baumgartner JC, Xia T, Siqueira JF Jr. Prevalence of selected bacterial named species and uncultivated phylotypes in endodontic abscesses from two geographic locations. J Endod 2006;32:1135-8. 